\title{
Chloroquine potentiates the anticancer effect of sunitinib on renal cell carcinoma by inhibiting autophagy and inducing apoptosis
}

\author{
MAN-LI LI ${ }^{1 *}$, YOU-ZHI XU ${ }^{2 *}$, WEN-JIE LU ${ }^{2 *}$, YONG-HUAI LI ${ }^{3}$, SHI-SHENG TAN $^{4}$, \\ HONG-JUN LIN ${ }^{1}$, TIAN-MING WU ${ }^{1}$, YAN LI ${ }^{1}$, SI-YING WANG ${ }^{2}$ and YING-LAN ZHAO ${ }^{1}$
}

\begin{abstract}
${ }^{1}$ Cancer Center, West China Hospital, West China Medical School, Sichuan University and Collaborative Innovation Center, Chengdu, Sichuan 610041; ${ }^{2}$ School of Basic Medical Sciences, Anhui Medical University, Hefei, Anhui 230032; ${ }^{3}$ Department of Respiratory Disease, The First Affiliated Hospital of Anhui Medical University, Hefei, Anhui 230022;

${ }^{4}$ Department of Oncology, Guizhou People's Hospital, Guiyang, Guizhou 510002, P.R. China
\end{abstract}

Received October 21, 2015; Accepted September 22, 2017

DOI: $10.3892 / \mathrm{ol} .2017 .7635$

\begin{abstract}
Sunitinib based adjuvant chemotherapy combined with chloroquine (CQ) for the treatment of renal cell carcinoma (RCC) is in clinical trials; however, its anti-RCC effect and the mechanism remain unclear. In the present study, the anti-RCC effect of sunitinib with CQ and the underlying mechanism was investigated. An MTT assay demonstrated that CQ enhanced the proliferation inhibitory effect of sunitinib against the OS-RC-2 RCC cell line. CQ inhibited sunitinib-induced autophagy in OS-RC-2, which was evidenced by the inhibition of autophagic vacuoles, acidic vesicular organelle formation, light chain 3 (LC3)-II recruitment to the autophagosomes and the conversion of LC3-I to LC3-II, as induced by sunitinib. The inhibition of autophagy by CQ enhanced sunitinib-induced apoptosis, which was characterized by the activation of caspase-3, caspase-9, Bcl-2 and p53. Additionally, the exposure of OS-RC-2 cells to CQ and sunitinib resulted in the inhibition of AKT, tuberous sclerosis complex 2, mechanistic target of rapamycin and p70 ribosomal S6 kinase, which are associated with cell proliferation. In in vivo study, a combination of sunitinib with CQ in mice significantly reduced OS-RC-2 cell xenograft growth compared with the sunitinib alone group. In conclusion, the present study demonstrated that CQ may enhance the anti-RCC effect of sunitinib by inhibiting the autophagy induced by sunitinib, and enhance the rate of
\end{abstract}

Correspondence to: Professor Ying-Lan Zhao, Cancer Center, West China Hospital, West China Medical School, Sichuan University and Collaborative Innovation Center, 17, 3rd Section, Ren Min South Road, Chengdu, Sichuan 610041, P.R. China

E-mail: zhaoyinglan@scu.edu.cn

\section{*Contributed equally}

Abbreviations: RCC, renal cell carcinoma; CQ, chloroquine; $\Delta \psi \mathrm{m}$, mitochondrial membrane potential; TUNEL, terminal deoxynucleotidyl transferase-mediated dUTP nick end labeling; AVOs, acidic vesicular organelles; FCM, flow cytometry

Key words: sunitinib, chloroquine, autophagy, apoptosis apoptosis. Inhibiting cell proliferation may also serve a role in the synergistic antitumor effect of sunitinib and CQ. These data suggest that combination therapy of sunitinib with $\mathrm{CQ}$ may be a promising strategy for adjuvant chemotherapy in RCC.

\section{Introduction}

Renal cell carcinoma (RCC) was the third most common urological cancer worldwide in 2010 (1). Despite the use of multimodal therapy (chemotherapy, radiation therapy and surgery), the long-term disease-free survival rate for patients with RCC remains low (2). Sunitinib is considered the standard of care for the first-line therapy of advanced clear cell RCC $(3,4)$. However, the majority of patients eventually develop resistance to sunitinib therapy, resulting in therapy failure. To improve the therapy efficiency of sunitinib, additional studies are currently being undertaken, including trials of sunitinib in combination with chemotherapy or molecular targeted agents (5). Although a number of combination therapies with sunitinib are being tested in clinical trials, there are further novel chemotherapy combinations to be explored (6).

Chloroquine (CQ) has previously been considered as a potential anti-cancer agent and a chemo-sensitizer in combination with anti-cancer drugs; it has been demonstrated to inhibit cell growth and/or induce cell death in various types of cancer $(7,8)$. At present, sunitinib-based adjuvant chemotherapy in combination with CQ for the treatment of RCC is in a phase I clinical trial (9). However, the understanding of its antitumor effect and mechanism remain incomplete.

Autophagy is the self-digestive process of the lysosomal degradation of mature proteins and organelles to maintain cellular homeostasis (10). A number of antineoplastic therapies have been observed to induce autophagy in human cancer cell lines, and autophagy induced by chemotherapy is considered a mechanism of resistance to therapy-mediated cell death $(11,12)$. CQ may act as an autophagy inhibitor by interfering with lysosomal acidification to block the autophagic process at the final step, which may enhance the antitumor effect of chemotherapy and induce cell apoptosis (13). Based on these observations, we hypothesized that sunitinib may induce autophagy in 
RCC, and CQ may enhance its antitumor effect by inhibiting autophagy. To the best of our knowledge, there are no studies concerning the autophagic potency of sunitinib on RCC and the molecular mechanisms of the potential synergistic effect of sunitinib and CQ.

In the present study, the combination efficiency of sunitinib with CQ was investigated in vitro and in vivo, and the underlying mechanism of their synergistic effect was examined. These results demonstrated that CQ may enhance the antitumor effect of sunitinib by inhibiting the autophagy induced by sunitinib, and enhance the rate of apoptosis, which may be a promising strategy for adjuvant chemotherapy in RCC.

\section{Materials and methods}

Cell culture. The OS-RC-2 human RCC cell line was purchased from the Cell Bank of Type Culture Collection of Chinese Academy of Sciences (Shanghai, China). The cells were cultured in RPMI-1640 media containing 10\% fetal bovine serum (Gibco; Thermo Fisher Scientific, Inc., Waltham, MA, USA) and $1 \%$ penicillin and streptomycin in humidified conditions with $5 \% \mathrm{CO}_{2}$ at $37^{\circ} \mathrm{C}$.

Materials. Sunitinib, CQ and all fluorescent dyes were purchased from Sigma-Aldrich; Merck KGaA (Darmstadt, Germany). The primary antibodies against Bcl-2 (sc-492), Bax (sc-70407) and $\beta$-actin (sc-47778) were purchased from Santa Cruz Biotechnology, Inc (Dallas, TX, USA). Primary antibodies against Beclin-1 (ab62557), autophagy related 5/12 (Atg5/12; ab78073) were purchased from Abcam (Cambridge, MA, USA). Primary antibodies against light chain 3 (LC3) I/II (no. 4108), p53 (no. 9282), caspase-3 (no. 9662), caspase-9 (no. 9508), phosphorylation of histone H3 (no. 9711), phosphoinositide 3-kinase (PI3K; no. 4255), Akt (no. 4685), phosphorylated (p)-Akt (no. 4058), tuberous sclerosis complex 2 (TSC2; no. 4308), p-TSC2 (no. 3617), p-mechanistic target of rapamycin (p-mTOR; no. 5536) and mTOR (no. 4517), p70 ribosomal S6 kinase (p70S6K; no. 2708) and p-p70S6K (no. 9204) were purchased from Cell Signaling Technology, Inc. (Danvers, MA, USA).

Cell viability assay. The cell viability was measured using an MTT assay, as previously described (14). Briefly, cells were seeded at $3-4 \times 10^{3}$ cells/well in a 96 -well plate, and treated with sunitinib and CQ for $48 \mathrm{~h}$. Then, $20 \mu \mathrm{l}$ MTT solutions $(5 \mathrm{mg} / \mathrm{ml})$ were added to each well for an additional $2 \mathrm{~h}$ at $37^{\circ} \mathrm{C}$. Following removal of the culture medium, dimethyl sulfoxide was added (200 $\mu \mathrm{l} /$ well) and the optical density was measured at $570 \mathrm{~nm}$ with a microplate reader.

Colony formation assay. A total of $1 \times 10^{3}$ cells/well were seeded in 6-well plates and treated with sunitinib and $25 \mu \mathrm{M} \mathrm{CQ}$. Subsequent to an additional incubation for 14 days, the cells were stained with $0.2 \%$ crystal violet for $10 \mathrm{~min}$ at room temperature, and the number of colonies in each well were counted.

Electron microscopy. Electron microscopy was performed to detect the induction of autophagic morphology. Following treatment with $25 \mu \mathrm{M} \mathrm{CQ}$ and $10 \mu \mathrm{M}$ sunitinib for $48 \mathrm{~h}$, cells were fixed with a solution containing $3 \%$ glutaraldehyde plus
$2 \%$ paraformaldehyde in $0.1 \mathrm{M}$ cacodylate buffer ( $\mathrm{pH} 7.3$ ) for $60 \mathrm{~min}$. The cells were then post-fixed in $1 \% \mathrm{OsO}_{4}$ buffer for $1 \mathrm{~h}$ on ice and subjected to transmission electron microscopy analysis at room temperature for $3 \mathrm{~h}$, as previously described (15).

Detection of acidic vesicular organelles. A total of $1 \times 10^{5}$ cells/well were plated in 6 -well plates. Following treatment with $25 \mu \mathrm{M}$ CQ and $10 \mu \mathrm{M}$ sunitinib for $48 \mathrm{~h}$, the cells were stained with $1 \mu \mathrm{g} / \mathrm{ml}$ acridine orange for $15 \mathrm{~min}$, washed with PBS and examined under a fluorescence microscope (Leica Microsystems, Wetzlar, Germany).

Determination of mean red:green fluorescence ratio with acridine. Cells $\left(1 \times 10^{5}\right.$ cells/well) were stained with $0.5 \mathrm{mg} / \mathrm{l}$ acridine orange for $10 \mathrm{~min}$, removed from the plate with trypsin-EDTA, and collected in phenol red-free growth medium. The number of red-fluorescing $(650 \mathrm{~nm})$ cells were measured with a FACSCalibur flow cytometer (BD Biosciences, San Diego, CA, USA).

Monodansylcadaverine (MDC) staining. Following treatment with $25 \mu \mathrm{M}$ CQ and $10 \mu \mathrm{M}$ sunitinib for $48 \mathrm{~h}$, the culture medium was replaced with fresh medium containing $0.05 \mathrm{mM}$ MDC and the cells were incubated at $37^{\circ} \mathrm{C}$ for $30 \mathrm{~min}$ in the dark. The cells were then washed three times with PBS and images were captured with fluorescence microscopy.

Transient transfection. OS-RC-2 cells were plated at a density of $1 \times 10^{5}$ on a coverslip and cultured until they reached $60 \%$ confluence. Then, the cells were transfected with an enhanced green fluorescent protein-LC3 (pEGFP-LC3) plasmid using Lipofectamine 2000 (Invitrogen; Thermo Fisher Scientific, Inc.) according to the manufacturer's protocol. When cells reached $90 \%$ confluence, drugs were added into the culture medium. GFP-LC3 fluorescence was then observed with fluorescence microscopy.

Immunofluorescence analysis. Following treatment with $25 \mu \mathrm{M}$ CQ and $10 \mu \mathrm{M}$ sunitinib for $48 \mathrm{~h}$, cells were fixed with methanol for $5 \mathrm{~min}$ on ice, and then washed with PBS for $10 \mathrm{~min}$. The cells were incubated with an LC3 antibody for $1 \mathrm{~h}$ at $37^{\circ} \mathrm{C}$, followed by incubation with a fluorescein isothiocyanate-conjugated secondary antibody, and staining with $1 \mu \mathrm{g} / \mathrm{ml}$ Hoechst 33342 for $1 \mathrm{~h}$ at $37^{\circ} \mathrm{C}$. The change in LC3 distribution was examined using a laser scanning confocal microscope (Leica Microsystems GmbH).

Western blot analysis. Following treatment with $25 \mu \mathrm{M} C \mathrm{CQ}$ and $10 \mu \mathrm{M}$ sunitinib for $48 \mathrm{~h}$, the cells were washed with PBS and lysed in radioimmunoprecipitation buffer $[150 \mathrm{mM} \mathrm{NaCl}$, $1.0 \%$ NP-40, $0.5 \%$ sodium deoxycholate, $0.1 \%$ SDS, $50 \mathrm{mM}$ Tris (pH 8.0), $1 \%$ pentylmethylsulfonyl fluoride and $0.1 \%$ cocktail (CT)]. The protein concentration in the cell lysates was measured using a Bio-Rad protein assay (Bio-Rad Laboratories, Inc., Hercules, CA, USA). Equal amounts of protein $(100 \mu \mathrm{g})$ were subjected to SDS-PAGE (10-15\% gels) and transferred onto polyvinylidene difluoride membranes. Subsequent to blocking with $5 \%$ non-fat milk at room temperature for $1 \mathrm{~h}$, incubation with the primary (1:1,000-1:2,000 dilution according to recommendations from antibodies procotol, overnight at $4^{\circ} \mathrm{C}$ ) 
and secondary antibodies: Anti-mouse IgG/horseradish peroxidase (HRP)-conjugated (cat no. ZDR-5307; ZSGB-BIO, Beijing, China) and anti-rabbit IgG/HRP-conjugated (cat no. BA1055; Boster Biotechnology Co., Ltd., Wuhan, China), the reactive band was identified using an enhanced chemiluminescence substrate with HRP (Amersham; GE Healthcare, Chalfont, UK).

Flow cytometry (FCM) for apoptosis detection and mitochondrial membrane potential assay. Following treatment with $25 \mu \mathrm{M}$ CQ and $10 \mu \mathrm{M}$ sunitinib for $48 \mathrm{~h}$, the cells were collected, suspended in $50 \mu \mathrm{g} / \mathrm{ml}$ propidium iodide for 10 min and analyzed using a FACSCalibur flow cytometer. Additionally, the mitochondrial membrane potential $(\Delta \psi \mathrm{m})$ and the mitochondrial permeability transition were determined by the retention of Rh123 dye. The cells were incubated with Rh123 $(5 \mathrm{~g} / \mathrm{ml})$ at $37^{\circ} \mathrm{C}$ for $30 \mathrm{~min}$, in the dark. Following two washes, the cells were analyzed with FCM or observed under an inverted fluorescence microscope (Leica Microsystems $\mathrm{GmbH}$ ).

In vivo study. Female BALB/c nude mice (6-8 weeks old, 18-22 g; Beijing Animal Center, Beijing, China) were used in the present study. The total numbers of mice used in the present study was 160 . The mice were maintained under controlled conditions at $21^{\circ} \mathrm{C}, 55 \%$ humidity, on a $12 \mathrm{~h}$ light /dark cycle and had food and water available ad libitum. The protocol was approved by the Animal Experimental Ethics Committee of the State Key Laboratory of Biotherapy, Sichuan University (Sichuan, China). OS-RC-2 cells $\left(5 \times 10^{6}\right)$ were subcutaneously injected into the hind flank of the mice. When the volume of the tumors reached $100 \mathrm{~mm}^{3}$, the mice were randomized into the vehicle control or treatment groups. Mice were administered with CQ (20 mg/kg/day) intravenously, sunitinib (20 or $40 \mathrm{mg} / \mathrm{kg} / \mathrm{day}$ ) orally or co-treatment with CQ and sunitinib for 28 days. Tumor growth and body weight were measured every 3 days during the treatment. Tumor volume was calculated using the following formula: Tumor volume $\left(\mathrm{mm}^{3}\right)=0.52$ $\mathrm{x} a \mathrm{x} b^{2}$, where $a$ is the length and $b$ is the width.

Terminal dexynucleotidyl transferase-mediated dUTP nick end labeling (TUNEL) assay. A TUNEL assay was performed to measure cellular apoptosis in vivo, according to the manufacturer's protocol (Promega Corporation, Madison, WI, USA). The TUNEL-positive cells were identified using a laser scanning confocal microscope (Leica Microsystems GmbH).

Immunohistochemistry analysis. The phosphorylation of histone H3 and LC3 were detected using immunohistochemistry analysis. Paraffin sections (4- $\mu \mathrm{m}$ thick) of the tumor tissue were incubated overnight at $4^{\circ} \mathrm{C}$ with phosphorylation of histone H3 (1:50) and LC3 polyclonal antibodies (1:100), followed by incubation at $4^{\circ} \mathrm{C}$ for $2 \mathrm{~h}$ with a biotinylated secondary antibody (HRP-anti rabbit $\mathrm{IgG}$; cat no. BA1055; 1:300; Boster Biotechnology Co., Ltd., Wuhan, China) for $1 \mathrm{~h}$. Diaminobenzidine was used to visualize the staining.

Statistical analysis. All results were expressed as the means \pm standard deviation. Statistical analysis was performed using SPSS v.13.0 software (SPSS, Inc., Chicago, IL, USA). A post hoc test with GraphPad Prism 5 (San Diego, California) analysis of variance was used for the comparison of data.
$\mathrm{P}<0.05$ was considered to indicate a statistically significant difference.

\section{Results}

Inhibitory effects of sunitinib on cell proliferation are enhanced by $C Q$ treatment in RCC cell lines. In order to investigate the proliferation inhibitory properties of sunitinib and $\mathrm{CQ}$, the cell viability was determined using an MTT assay. As demonstrated in Fig. 1A, OS-RC-2 cell growth was effectively inhibited by sunitinib in a concentration-dependent manner; $\mathrm{CQ}$ at concentrations of $25 \mu \mathrm{M}$ exhibited almost no inhibitory effect on cell proliferation following $24 \mathrm{~h}$ treatment (data not shown), so was selected for the subsequent experiments. The inhibition rates were 25 and $35 \%$ when the cells were treated with 3.75 and $7.5 \mu \mathrm{M}$ sunitinib, respectively, whereas the inhibition rate increased to 34 and $58 \%$ following co-treatment.

A colony formation assay was used to further determine whether CQ was able to enhance the proliferation inhibitory effect. The results clearly demonstrated that CQ significantly enhanced the inhibitory effect of sunitinib exposure on clone formation (Fig. 1B). These results suggested that CQ may enhance sunitinib-inhibited cell proliferation in OS-RC-2 cells.

$C Q$ inhibits sunitinib-induced autophagy in OS-RC-2 cells. To examine the mechanism responsible for the CQ-enhanced inhibitory effect of sunitinib on proliferation, whether sunitinib may induce autophagy in OS-RC-2 cells and whether CQ may inhibit sunitinib-induced autophagy were examined. Firstly, transmission electron microscopy analysis was performed following $10 \mu \mathrm{M}$ sunitinib treatment of the OS-RC-2 cells for 48 h. As demonstrated in Fig. 2A, numerous autophagosomes were observed in the cytoplasm of sunitinib-treated cells, whereas the number of membrane-bound vacuoles decreased when cells were treated with sunitinib and CQ.

To evaluate if the formation of membrane-bound vacuoles was an autophagic response upon sunitinib treatment, the formation of the acidic vesicular organelles (AVOs) was analyzed with acridine orange staining. As indicated by Fig. 2B, sunitinib treatment resulted in the formation of yellow-orange AVOs in OS-RC-2 cells. Following CQ and sunitinib treatment, the number of yellow-orange AVOs increased.

To further quantify the formation of AVOs, FCM analysis was performed. The results demonstrated that the percentage of AVOs positive cells increased to $48.0 \%$ following sunitinib treatment, whereas it was $0.76 \%$ in control cells. Subsequent to CQ treatment, the AVOs formation increased to $56.88 \%$; the results from FCM analysis were consistent with the results from fluorescence microscopy (Fig. 2C).

MDC staining was used to further detect AVOs; co-treatment with CQ and sunitinib increased the number of MDC-labeled green fluorescent particles compared with sunitinib alone (Fig. 2D). Concurrently, the typical characteristics of autophagy were also observed; the formation of autophagic vacuoles was confirmed by GFP-LC3 distribution (Fig. 2E). Co-treatment increased the extent of GFP-LC3 localization induced by sunitinib. The formation of autophagosomes was also evaluated using immunofluorescence, and GFP puncta were detected (Fig. 2F). Taken together, these results demonstrate that sunitinib may induce autophagy and CQ may inhibit this effect. 


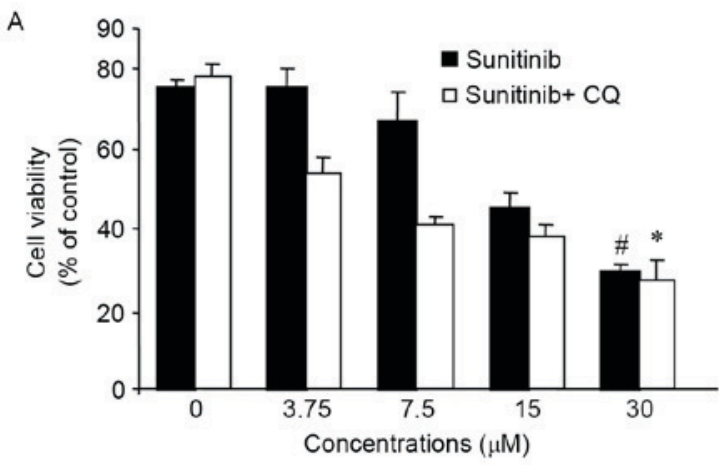

B

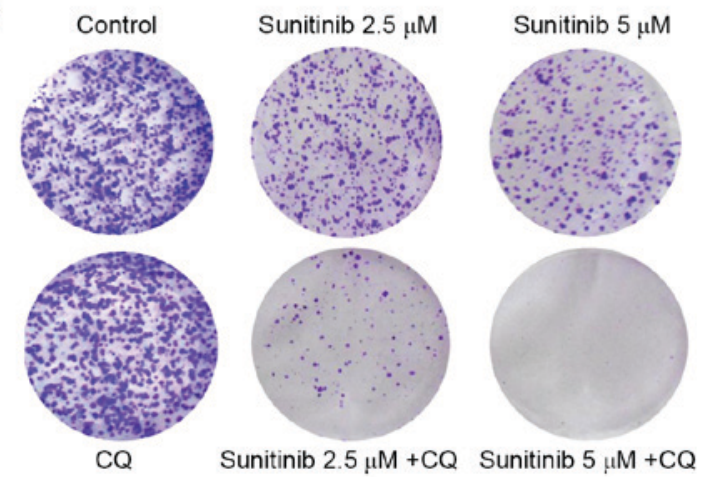

Figure 1. Combination of sunitinib with CQ inhibited the proliferation of renal cancer cells. (A) Cell viability was determined by an MTT assay following treatment with sunitinib alone, or in combination with $25 \mu \mathrm{M} \mathrm{CQ}$. (B) Effect of sunitinib and CQ on the colony formation of OS-RC-2 cells over 2 weeks. The data are representative of $\geq 3$ independent experiments. ${ }^{*} \mathrm{P}<0.05$ compared with $0 \mu \mathrm{M}$ sunitinib; ${ }^{\mathrm{P}}<0.05$ compared with $0 \mu \mathrm{M}$ sunitinib $+\mathrm{CQ}$. $\mathrm{CQ}$, chloroquine.

Sunitinib increases the expression of autophagy-associated proteins. In order to further confirm the sunitinib-induced autophagy, the expressions of autophagy-associated proteins were examined using western blot analysis. The increased conversion of LC3-I to LC3-II is considered to be an autophagosomal marker due to its localization and aggregation on autophagosomes. It was demonstrated that native LC3-II accumulation was increased in sunitinib-treated cells, and the expression of other proteins, including Atg5-Atg12 conjugations and Beclin-1, also increased (Fig. 3A). Concurrently, the sunitinib-induced upregulation of autophagy-associated proteins was increased by co-treatment.

Combining sunitinib with CQ treatment induces apoptosis. To determine whether sunitinib- and CQ-treated tumor cells undergo apoptosis, an FCM apoptosis assay was performed. The rate of apoptosis was 26.5 and $43.7 \%$, respectively, when cells were treated with 5 and $10 \mu \mathrm{M}$ sunitinib. However, the rate of apoptosis increased to 39.2 and $78.3 \%$, respectively, for co-treated cells (Fig. 3B). Therefore, it was concluded that CQ may improve the rate of apoptosis induced by sunitinib. Additionally, the change in $\Delta \psi \mathrm{m}$ was detected using an FCM assay. The loss of $\Delta \psi \mathrm{m}$ was 13.3 and $22.3 \%$, following treatment with 5 and $10 \mu \mathrm{M}$ sunitinib, respectively, whereas the loss of $\Delta \psi \mathrm{m}$ increased to 21.1 and $90.8 \%$ subsequent to co-treatment (Fig. 3C). Similar results were obtained from staining the mitochondria membrane (Fig. 3D). A total of
$>50 \%$ of the cells were intensely green following CQ and sunitinib co-treatment.

In addition, western blot analysis revealed a potential molecular mechanism of sunitinib-induced apoptosis as the levels of cleaved caspase- 3 and caspase- 9 increased in a concentration-dependent manner. A decrease of Bcl-2 and increases of Bax and p53 were also observed (Fig. 3E). These phenomena were more apparent following co-treatment. In addition, to explore whether sunitinib-induced apoptosis was specifically associated with the caspase family, a caspase inhibitor, Z-VAD-FMK, was administered to cells undergoing sunitinib-induced apoptosis. As demonstrated in Fig. 3F, the apoptosis rate was 20 vs. 14\% with $5 \mu \mathrm{M}$ sunitinib, and 48 vs. $22 \%$, with $10 \mu \mathrm{M}$ sunitinib, with and without $2 \mu \mathrm{M}$ Z-VAD-FMK, respectively. Similar results were obtained following co-treatment with CQ. Therefore, apoptosis induced by sunitinib may be partly reversed by a pan-caspase inhibitor, Z-VAD-FMK.

Sunitinib induced autophagy by inhibiting the Akt/mTOR/p70S6K signaling pathway in OS-RC-2 cells. As the $\mathrm{Akt} / \mathrm{mTOR} / \mathrm{p} 70 \mathrm{~S} 6 \mathrm{~K}$ pathway is the main regulatory pathway that negatively regulates autophagy, whether sunitinib and CQ treatment induced an alteration in this pathway was examined using a western blotting assay. The results demonstrated that sunitinib decreased the phosphorylation of Akt, TSC2, mTOR and p70S6K, whereas the total levels of PI3K, Akt, TSC2, mTOR and p70S6K were not altered (Fig. 4A). These results suggest that the upstream pathway of the Akt/mTOR/p70S6K pathway was inhibited by sunitinib treatment, and that sunitinib may induce autophagy through this pathway. In addition, the combination of CQ and sunitinib treatment enhanced the suppression of this pathway in a concentration-dependent manner.

In vivo antitumor activity of combined treatment with sunitinib and $C Q$. In vivo study was performed to validate the antitumor activity of sunitinib and CQ. The results demonstrated that the inhibition of tumor volume was $47 \%(20 \mathrm{mg} / \mathrm{kg}$ ) and $56 \%$ (40 $\mathrm{mg} / \mathrm{kg}$ ) following sunitinib treatment compared with the control group. However, the inhibition of tumor volume reached 64 and 73\%, respectively, following co-treatment with CQ (Fig. 4B). No loss of body weight was observed during sunitinib and CQ treatment (Fig. 4C). In addition, the effect on the apoptosis and proliferation of xenograft tumors in mice was evaluated. As demonstrated in Fig. 4D, an increase in the levels of nuclear green fluorescence was observed in the co-treatment group compared with sunitinib treatment alone in the TUNEL assay. In the in vivo proliferation assay, the appearance of brown spots, which indicated the phosphorylation of histone $\mathrm{H} 3$, was reduced following sunitinib treatment compared with the control, and decreased further following co-treatment with sunitinib and CQ (Fig. 4E). Therefore, these results suggested that co-treatment enhanced cell apoptosis and inhibited cell proliferation. The changes in autophagy in mice tumors following drug treatment were also investigated. Immunohistochemistry analysis revealed that the brown punctate staining, which represented the autophagic marker of LC3-II, was increased in the co-treatment group compared with the sunitinib group (Fig. 4F). Taken together, these results suggested that co-treatment may improve the antitumor activity of sunitinib though the inhibition of autophagy. 
A

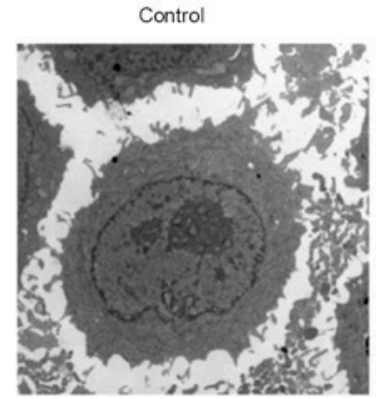

B
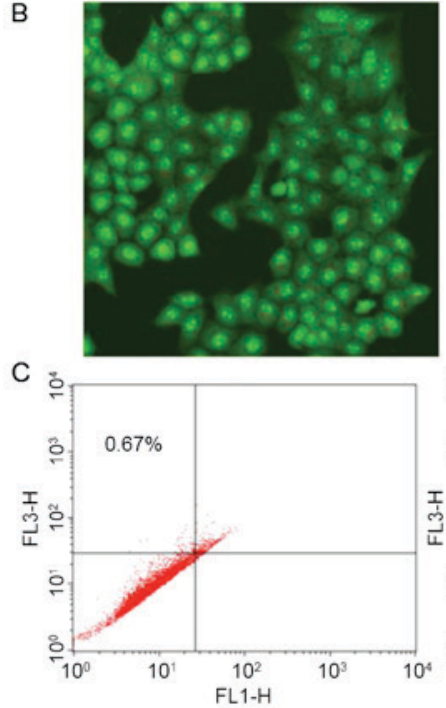

D

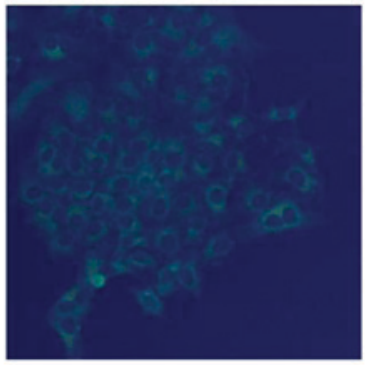

E

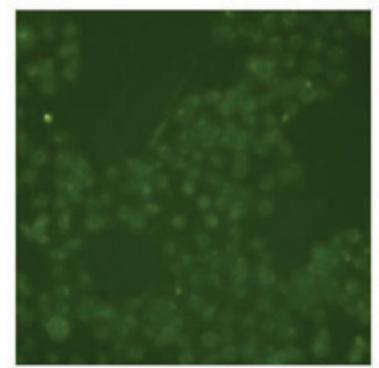

$\mathrm{F}$

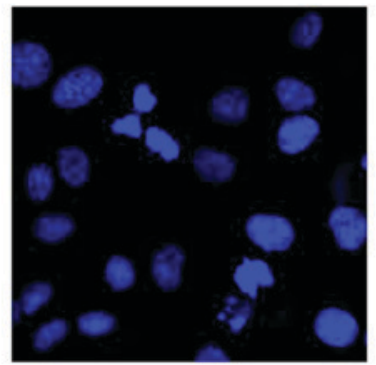

$\mathrm{CQ}$
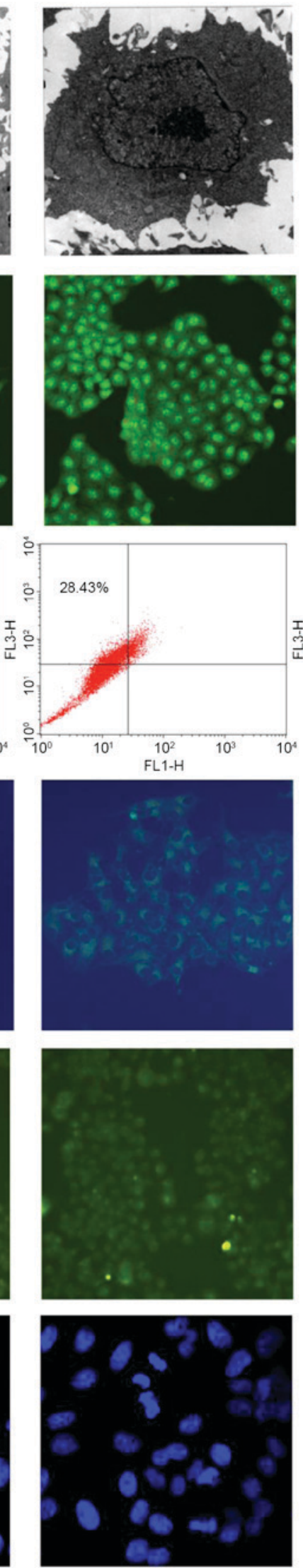

Sunitinib
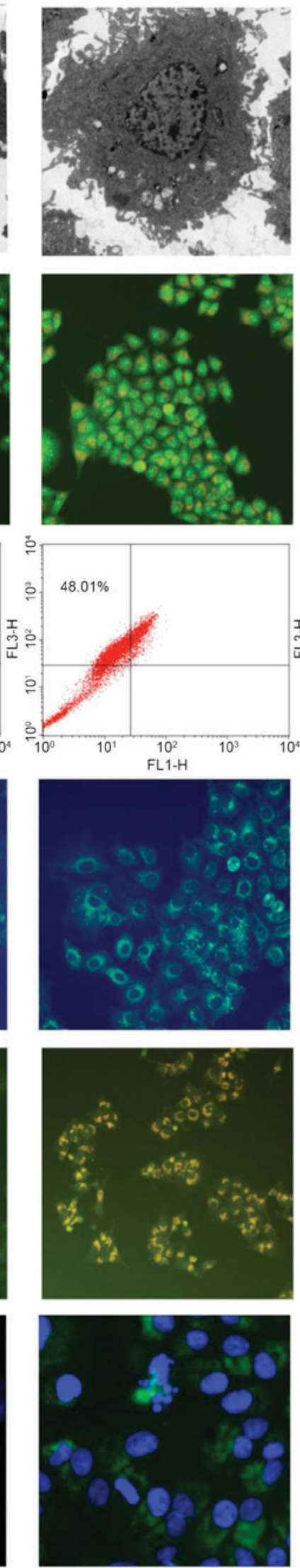

Sunitinib+CQ
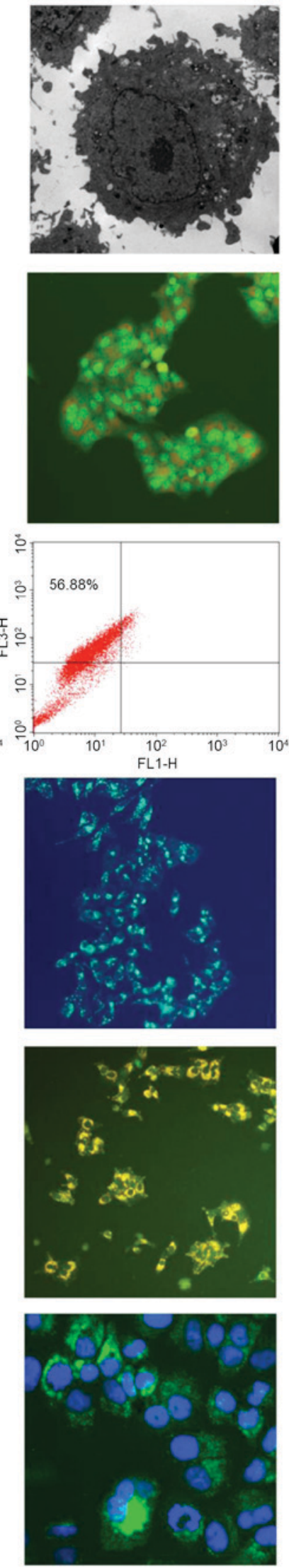

Figure 2. CQ inhibited sunitinib-induced autophagy in OS-RC-2 cells. (A) Representative electron micrographs demonstrate ultra-structural alterations following $10 \mu \mathrm{M}$ sunitinib and $25 \mu \mathrm{M} \mathrm{CQ}$ treatment alone or in combination (x400). (B) Cells were stained with $1 \mathrm{mg} / \mathrm{ml}$ acridine orange and visualized by fluorescence microscopy (x200). (C) Acidic vesicular organelles were quantified with fluorescence-activated cell sorting analysis. (D) Cells were stained with monodansylcadaverine and the fluorescence was observed (magnification, x200). (E) OS-RC-2 cells were transiently transfected with an enhanced green fluorescent protein-light chain 3 plasmid and treated with sunitinib and CQ (x50). (F) The fluorescence intensity of LC3 was detected following sunitinib and CQ treatment. CQ, chloroquine (x200). 

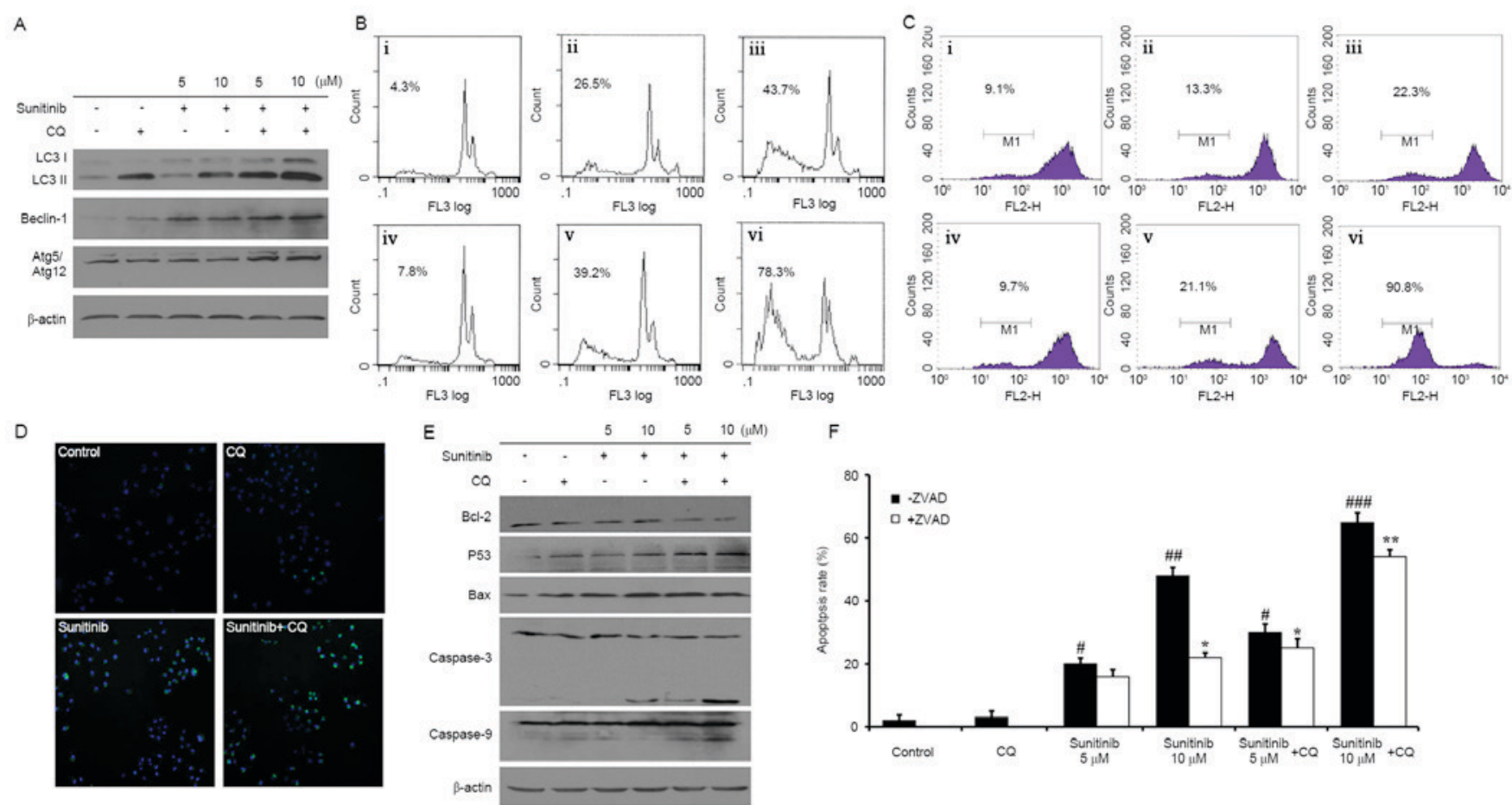

F
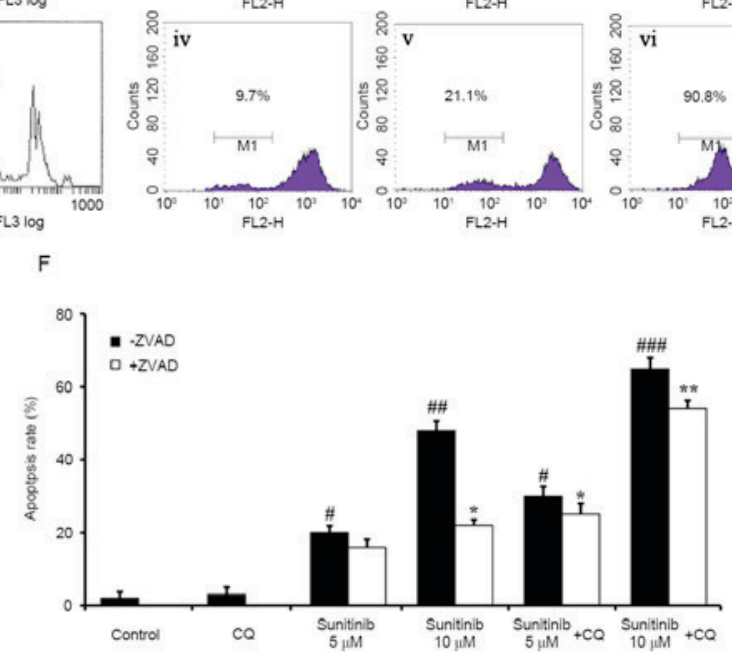

Figure 3. Expression of autophagy-associated proteins and sunitinib with CQ induced apoptosis in renal cancer cells. (A) Protein expression of LC3, Atg5, and Beclin-1 in OS-RC-2 cells treated with various treatment concentrations. (B) Flow cytometry was used to determine following propidium iodide staining. i, Control; ii, $5 \mu \mathrm{M}$ sunitinib; iii, $10 \mu \mathrm{M}$ sunitinib; iv, $25 \mu \mathrm{M}$ CQ; v, $5 \mu \mathrm{M}$ sunitinib + CQ; vi, $10 \mu \mathrm{M}$ sunitinib + CQ. (C) Mitochondrial permeability transition in OS-RC-2 cells following drug treatment. i, control; ii, $5 \mu \mathrm{M}$ sunitinib; iii, $10 \mu \mathrm{M}$ sunitinib; iv, $25 \mu \mathrm{M} \mathrm{CQ}$; v, $5 \mu \mathrm{M}$ sunitinib + CQ; vi, $10 \mu \mathrm{M}$ sunitinib + CQ (D) Microscopy images displaying the relative extent of fluorescence intensity, representing the extent of mitochondrial permeability following treatment with $10 \mu \mathrm{M}$ sunitinib and/or $25 \mu \mathrm{M}$ CQ. (E) Protein expression of Bcl-2, Bax, caspase-9 and caspase-3 in OS-RC-2 cells. (F) OS-RC-2 apoptosis was determined following treatment with $2 \mu \mathrm{M}$ Z-VAD-FMK. All data are representative of three independent experiments. " and ${ }^{\#,}, \mathrm{P}<0.05 ;{ }^{* *}$ and ${ }^{\# \#, ~} \mathrm{P}<0.01 ;{ }^{\# \# \#} \mathrm{P}<0.001$; "compared with control (-ZVAD); " compared with control (+ZVAD). CQ, chloroquine; LC3, light chain 3; Atg5, autophagy protein 5; Bax, Bcl-2-associated X.

\section{Discussion}

Sunitinib is an anticancer drug used for the treatment of RCC, and sunitinib in combination with CQ for the treatment of RCC is in clinical trials $(16,17)$. Understanding the underlying mechanism of sunitinib in combination with CQ is important for its future use in clinical therapy. To the best of our knowledge, the present study is the first to demonstrate that CQ may enhance the anti-RCC effect of sunitinib by inhibiting the autophagy induced by sunitinib.

Sunitinib is currently considered the standard of care for the first-line therapy of RCC (18). However, the vast majority of patients eventually develop resistance to sunitinib therapy $(4,19)$. Therefore, a complete understanding of the mechanisms of sunitinib action against RCC is critical in understanding and improving the treatment of this disease. Previously, autophagy has been demonstrated as an adaptive mechanism that contributes to tumor cell survival and resistance to therapy-induced apoptosis. There are a number of pharmacological mediators that can clinically induce autophagy. For example, temozolomide, 5-fluorouracil and tyrosine kinase inhibitors, including imatinib and dasatinib, have also been demonstrated to induce autophagy, which may decrease the anticancer efficiency of anticancer drugs (20). Therefore, there is interest in determining whether sunitinib induces autophagy in human RCC cells. In the present study, it was demonstrated that sunitinib may induce the formation of AVOs, GFP-LC3 punctuates and autophagosomes in OS-RC-2 cells. In addition, pathognomonic autophagy-associated genes, including LC3, Atg5 and BECN1, were increased following sunitinib treatment. Based on these results, it may be concluded that sunitinib induces autophagy in OS-RC-2 cells.

Autophagy is a temporary survival mechanism of cancer cells to adapt to the stressful conditions caused by anticancer therapies (12). Previous studies have revealed that autophagy inhibitors, including 3-methyladenine and CQ, may sensitize cancer cells to chemotherapy or radiation, and enhance the antitumor effect of anticancer drugs (21). CQ inhibits lysosome acidification, therefore blocking the late stages of autophagy, and is already clinically available as an autophagy inhibitor (7). Combinations of chemotherapeutic agents with CQ are in clinical trials against breast cancer, multiple myeloma, prostate cancer and other types of advanced tumor (22). The combination of sunitinib and CQ is in phase I clinical trials against RCC (23). In the present study, in vivo and in vitro antitumor assays demonstrated that CQ may significantly increase the anti-RCC activity of sunitinib. In addition, during the 28 days of treatment, CQ combined with sunitinib was well-tolerated by the mice and no adverse effects were observed. To gain insight into the molecular mechanism, the changes in autophagy were investigated. The results of the present study demonstrated that CQ increased the formation of AVOs, GFP-LC3 puncta and autophagosomes compared with sunitinib treatment alone in OS-RC-2 cells. The LC3, Atg5 and Beclin-1 protein expression increased following the 

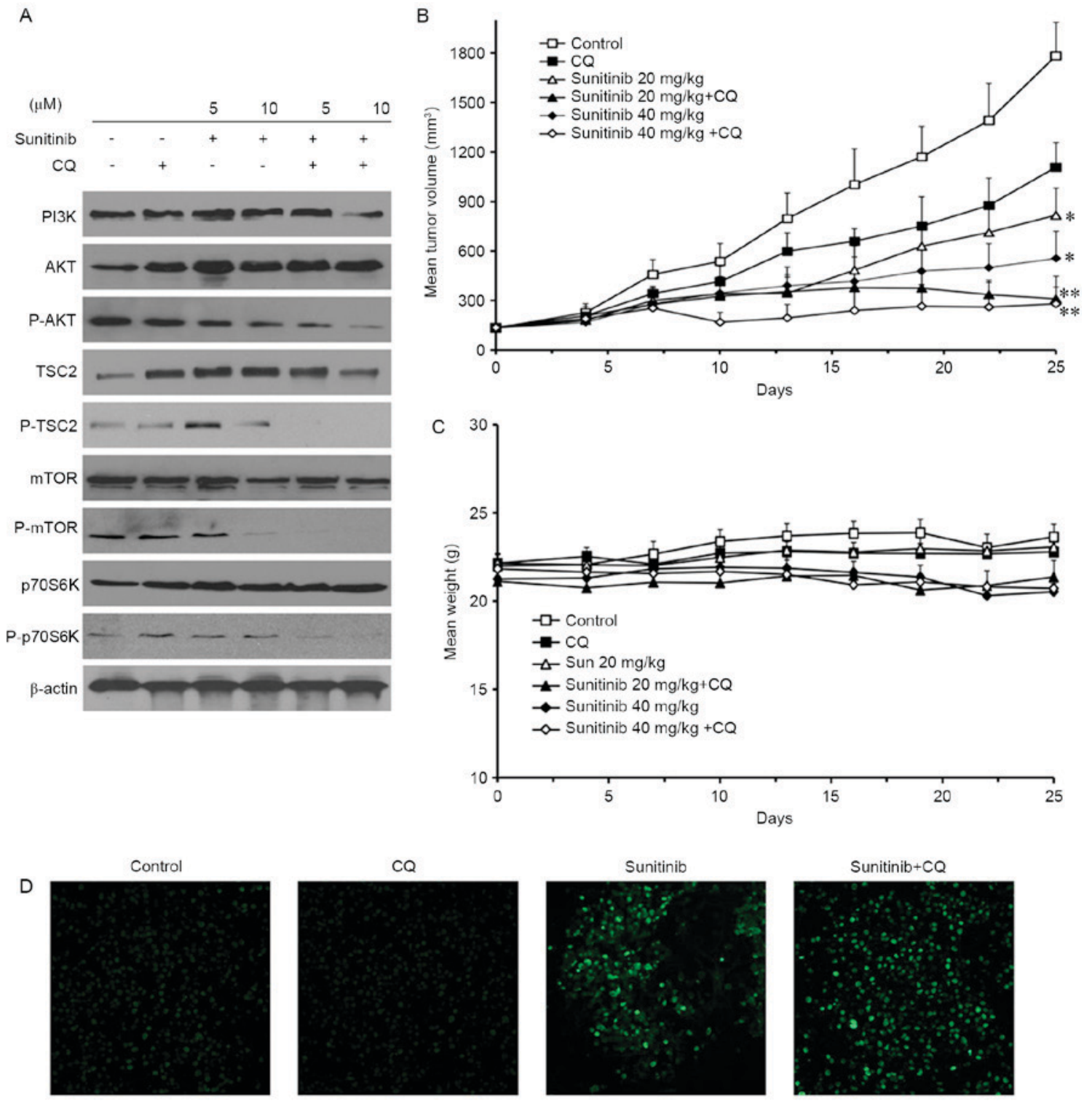

$\mathrm{E}$
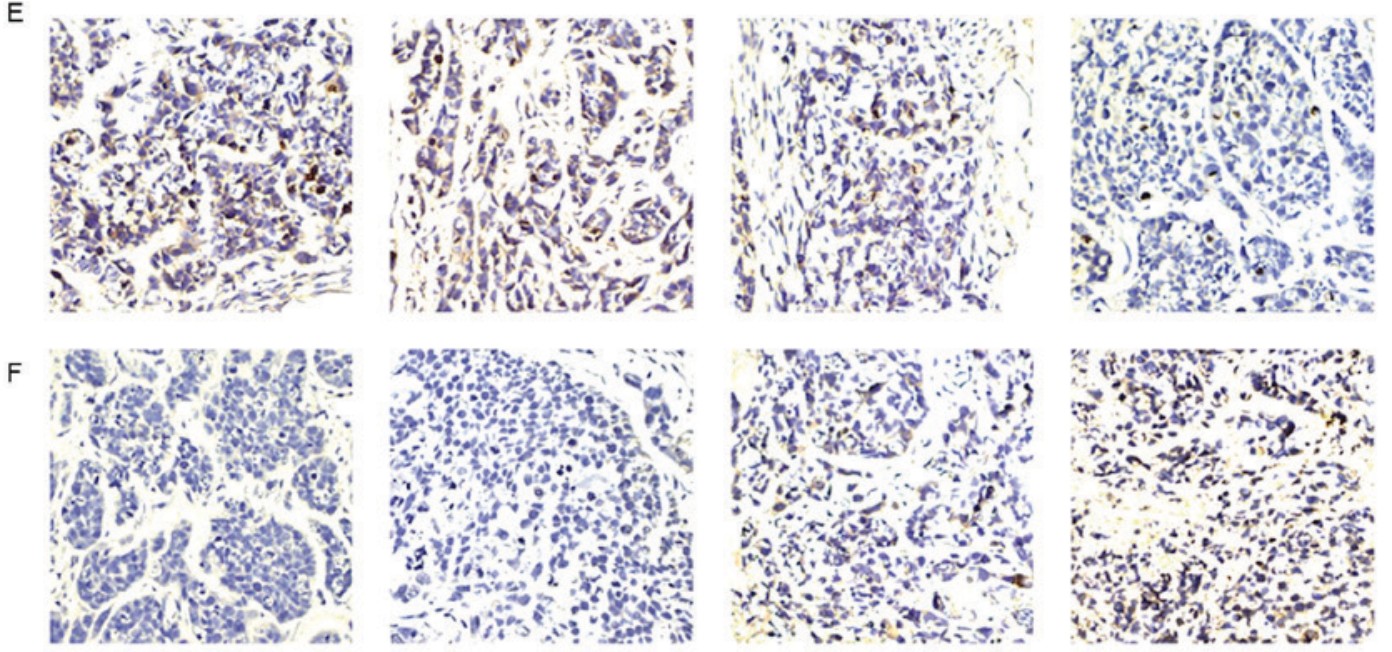

Figure 4. Sunitinib induces autophagy by inhibiting the Akt/mTOR/p70S6K signaling pathway, and may inhibit the growth of OS-RC-2 tumors in vivo. (A) Protein expression of PI3K, AKT, p-AKT, TSC2, p-TSC2, mTOR, p-mTOR, p70S6K and p-p70S6K in OS-RC-2 cells. (B) The inhibitory effect on the proliferation of OS-RC-2 cells established in nude BALB/c mice following CQ (20 mg/kg/day intravenously), sunitinib (40 mg/kg/day orally) or both. The data were expressed as the mean \pm SD. (C) Mouse body weight was not significantly altered following sunitinib and CQ co-treatment. (D) A terminal dexynucleotidyl transferase-mediated dUTP nick end labeling assay was performed to measure the induction of apoptosis. (E) Tumor cell proliferation in different groups was analyzed by the staining of phosphorylated histone H3. (F) Tumor cell autophagy in different groups was analyzed by LC3-II staining. Data represent the means $\pm \mathrm{SD}$ or are representative of $\geq 3$ independent experiments (magnification, $\mathrm{x} 200$ ). ${ }^{*} \mathrm{P}<0.05$ and ${ }^{* *} \mathrm{P}<0.01$ compared with control. PI3K, phosphoinositide 3-kinase; p-, phosphorylated; TSC2, tuberous sclerosis complex 2; mTOR, mechanistic target of rapamycin; p70S6K, p70S6 kinase; CQ, chloroquine; SD, standard deviation. 
co-treatment with sunitinib and CQ. Thus, it may be concluded that the autophagy induced by sunitinib was inhibited by CQ.

Autophagy is considered a survival mechanism in sunitinib-treated cells (24). The suppression of autophagy leads to apoptosis, thus enhancing the antitumor effect. The loss of $\Delta \psi \mathrm{m}$ is one of the hallmarks of apoptosis. In the mitochondrial-mediated apoptotic pathway, caspase family proteins caspase-3 and caspase-9 serve a central role. Co-treatment with sunitinib and CQ increased the levels of anti-apoptotic Bcl-2 and p53, and decreased the pro-apoptotic Bax protein expression. This result is in accord with a previous study, which reported that CQ may improve dasatinib-induced apoptosis (20). At present, the precise molecular mechanism that links autophagy and apoptosis is not clear. Autophagy and apoptosis may be induced in response to different cellular stresses, and the induction of autophagy/apoptosis may occur sequentially, simultaneously or in a mutually exclusive manner (25). The PI3K-Akt-mTOR signaling pathway is an important negative regulator of autophagy (26). In the present study, it was demonstrated that the phosphorylation of Akt, mTOR, TSC2 and p70S6K was decreased following sunitinib treatment, suggesting that sunitinib inhibited Akt-mTOR signaling and induced autophagy. However, according to the results of the present study, CQ also inhibited this pathway, which suggests that it may be associated with anti-tumor activity. In addition, apoptosis and autophagy are not mutually exclusive pathways; they have been observed to act in synergy or in opposition to each other. They share a number of the same molecular regulators. Therefore, the present study indicated that sunitinib may induce autophagy in vivo and in vitro as a defense mechanism.

To conclude, to the best of our knowledge, the present study demonstrated for the first time that CQ may enhance the anti-RCC effect of sunitinib by inhibiting autophagy, as well as enhancing the rate of apoptosis and inhibiting cell proliferation. A combination therapy of sunitinib with $\mathrm{CQ}$ may be a promising strategy for adjuvant chemotherapy in RCC.

\section{Acknowledgements}

The present study was supported by the National S\&T Major project (grant nos. 2011ZX09102-001-013 and 2012ZX09501001-003), the Project of the National Natural Sciences Foundation of China (grant nos. 81272459 and 81402947), the Natural Sciences Foundation of Anhui Province (grant no. 1508085QH162) and the Grants for Scientific Research of BSKY from Anhui Medical University (grant nos XJ201315 and XJ201509).

\section{References}

1. Chow WH, Dong LM and Devesa SS: Epidemiology and risk factors for kidney cancer. Nat Rev Urol 7: 245-257, 2010.

2. Xu K, Ding Q, Fang Z, Zheng J, Gao P, Lu Y and Zhang Y: Silencing of HIF-1alpha suppresses tumorigenicity of renal cell carcinoma through induction of apoptosis. Cancer Gene Ther 17: 212-222, 2009.

3. Zhao Y, Xue T, Yang X, Zhu H, Ding X, Lou L, Lu W, Yang B and He Q: Autophagy plays an important role in sunitinib-mediated cell death in H9c2 cardiac muscle cells. Toxicol App Pharmacol 248: 20-27, 2010.

4. Motzer RJ, Rini BI, Bukowski RM, Curti BD, George DJ, Hudes GR, Redman BG, Margolin KA, Merchan JR, Wilding G, et al: Sunitinib in patients with metastatic renal cell carcinoma. JAMA 295: 2516-2524, 2006.
5. Chairatvit K and Ngamkitidechakul C: Control of cell proliferation via elevated NEDD8 conjugation in oral squamous cell carcinoma. Mol Cell Biochem 306: 163-169, 2007.

6. Bello CL, Sherman L, Zhou J, Verkh L, Smeraglia J, Mount J and Klamerus KJ: Effect of food on the pharmacokinetics of sunitinib malate (SU11248), a multi-targeted receptor tyrosine kinase inhibitor: Results from a phase I study in healthy subjects. Anticancer Drugs 17: 353-358, 2006.

7. Sasaki K, Tsuno NH, Sunami E, Tsurita G, Kawai K, Okaji Y, Nishikawa T, Shuno Y, Hongo K, Hiyoshi M, et al: Chloroquine potentiates the anti-cancer effect of 5-fluorouracil on colon cancer cells. BMC Cancer 10: 370, 2010.

8. Solomon VR and Lee H: Chloroquine and its analogs: A new promise of an old drug for effective and safe cancer therapies. Euro J Pharmacol 625: 220-233, 2009.

9. Flanigan RC, Campbell SC, Clark JI and Picken MM: Metastatic renal cell carcinoma. Curr Trea Option Oncol 4: 385-390, 2003.

10. Meijer AJ and Codogno P: Regulation and role of autophagy in mammalian cells. Int J Biochem Cell Biol 36: 2445-2462, 2004.

11. Ng G and Huang J: The significance of autophagy in cancer. Mol Carcinogen 43: 183-187, 2005.

12. Notte A, Leclere L and Michiels C: Autophagy as a mediator of chemotherapy-induced cell death in cancer. Biochem Pharmacol 82: 427-434, 2011

13. Carew JS, Nawrocki ST and Cleveland JL: Modulating autophagy for therapeutic benefit. Autophagy 3: 464-467, 2007.

14. Xu Y, Lu W, Yang P, Peng W, Wang C, Li M, Li Y, Li G, Meng N, Lin H, et al: A small molecular agent YL529 inhibits VEGF-D-induced lymphangiogenesis and metastasis in preclinical tumor models in addition to its known antitumor activities. BMC Cancer 15: 525, 2015.

15. Asoro MA, Kovar D and Ferreira PJ: In situ transmission electron microscopy observations of sublimation in silver nanoparticles. ACS Nano 9: 7844-7852, 2013.

16. Nanus DM, Garino A, Milowsky MI, Larkin M and Dutcher JP: Active chemotherapy for sarcomatoid and rapidly progressing renal cell carcinoma. Cancer 101: 1545-1551, 2004.

17. Sunkara U, Walczak JR, Summerson L, Rogers T, Eisenberger M, Denmeade S, Pili R, Huff CA, Sinibaldi V and Carducci MA: A phase II trial of temozolomide and IFN-alpha in patients with advanced renal cell carcinoma. J Interf Cytok Res 24: 37-41, 2004.

18. Czarnecka AM, Szczylik C and Rini B: The use of sunitinib in renal cell carcinoma: Where are we now? Expert Rev Anticancer Ther 14: 983-999, 2014.

19. Motzer RJ, Hutson TE, Tomczak P, Michaelson MD, Bukowski RM, Oudard S, Negrier S, Szczylik C, Pili R, Bjarnason GA, et al: Overall survival and updated results for sunitinib compared with interferon alfa in patients with metastatic renal cell carcinoma. J Clin Oncol 27: 3584-3590, 2009.

20. Milano V, Piao Y,LaFortune T and de Groot J: Dasatinib-induced autophagy is enhanced in combination with temozolomide in glioma. Mol Cancer ther 8: 394-406, 2009.

21. Li J, Hou N, Faried A, Tsutsumi S and Kuwano H: Inhibition of autophagy augments 5-fluorouracil chemotherapy in human colon cancer in vitro and in vivo model. Euro J Cancer 46: 1900-1909, 2010.

22. Sui X, Chen R, Wang Z, Huang Z, Kong N, Zhang M, Han W, Lou F, Yang J, Zhang Q, et al: Autophagy and chemotherapy resistance: A promising therapeutic target for cancer treatment. Cell Death Dis 4: e838, 2013.

23. Levy JM and Thorburn A: Targeting autophagy during cancer therapy to improve clinical outcomes. Pharmacol Ther 131: 130-141, 2011.

24. Liu J, Fan L, Wang H and Sun G: Autophagy, a double-edged sword in anti-angiogenesis therapy. Med Oncol 33: 10, 2016.

25. Bhutia SK, Dash R, Das SK, Azab B, Su ZZ, Lee SG, Grant S, Yacoub A, Dent P, Curiel DT, et al: Mechanism of autophagy to apoptosis switch triggered in prostate cancer cells by antitumor cytokine melanoma differentiation-associated gene 7/interleukin-24. Cancer Res 70: 3667-3676, 2010.

26. Park EJ, Kim SY, Kim SH, Lee CR, Kim IS, Park JK, Lee SW, Kim BJ, Chun JN and Jeon JH: SK\&F 96365 induces apoptosis and autophagy by inhibiting Akt-mTOR signaling in A7r5 cells. Biochim Biophys Acta 1813: 2157-2164, 2011.

This work is licensed under a Creative Commons Attribution-NonCommercial-NoDerivatives 4.0 International (CC BY-NC-ND 4.0) License. 